\title{
Zoster infection after vaccination with the AstraZeneca COVID-19 vaccine: A case report
}

\section{Laurent Dupoirieux}

Department of Plastic Surgery, 74 avenue Jean Jaures 47200 Marmande, France.

Corresponding author: Laurent Dupoirieux MD, DMD, PhD, E-mail: 1dpx@yahoo.fr

\begin{abstract}
Herein, we present the case of a 55-year-old patient who developed a severe zoster reaction after receiving the AstraZeneca COVID-19 vaccine. Although zoster reactivation has already been observed with various vaccines, the extent and length of this reaction raise serious concerns. Hopefully, the outcome of this patient was favorable. The case of this patient, who could have only received a single dose of the vaccine, suggests that immunity after this type of vaccine vanishes rapidly and booster shots will be of crucial importance in the future.
\end{abstract}

Key words: Zoster reactivation; COVID-19; AstraZeneca vaccine

\section{INTRODUCTION}

COVID-19 vaccines were introduced during the global pandemic of SARS-CoV-2. Their purpose was to prevent the spread of the virus within the general population and to limit the number of serious cases. In the meantime, there is an increasing number of reports on the adverse effects. In this case, we present a case of severe zoster infection arising after injection of the AstraZeneca vaccine.

\section{CASE REPORT}

A 55-year-old British male living in France for several years was referred to my office for the excision of a painful lesion from the skin of one ear. During a clinical examination, he asked me to examine a lateral, thoracic, cutaneous eruption that appeared five days after vaccination with the AstraZeneca vaccine. The multiple crusty vesicles were typical of a zoster infection (Fig. 1). The patient mentioned no history of an allergy or previous zoster infection.

Treatment with Zovirax (aciclovir) at $200 \mathrm{mg}$ (5 tablets per day) was, thus, undertaken for seven days. Six days after the treatment, a marked improvement was observed, as the lesion dried. However, during the following two months, the patient complained of intense pain in the chest and arms. The pain finally settled without residual neuralgia.

A biological investigation showed no perturbation in the hemogram (leucocytes at $7.05 \mathrm{G} / \mathrm{L}$, with $30.93 \%$ of lymphocytes) and a normal coagulation blood test. A post-infection serologic test with an antibody dosage indicated a low level of anti-SARS-Cov-2 IgG, at $27.5 \mathrm{AU} / \mathrm{mL}$ (positivity threshold: $50 \mathrm{AU} / \mathrm{mL}$ ). The French health authority was informed about the incident. No decision was taken regarding the second jab.

\section{DISCUSSION}

The AstraZeneca vaccine was one of the first approved vaccines in the French vaccination campaign. Several dermatological lesions have been described after COVID-19 vaccination, yet most of them were local, delayed eruptions on the site of injection (urticaria, morbilliform eruptions) [1]. Varicella-zoster eruptions are less frequent but have been described as a potential side effect of the vaccine. The varicella-Zoster eruption observed in this patient was, however,

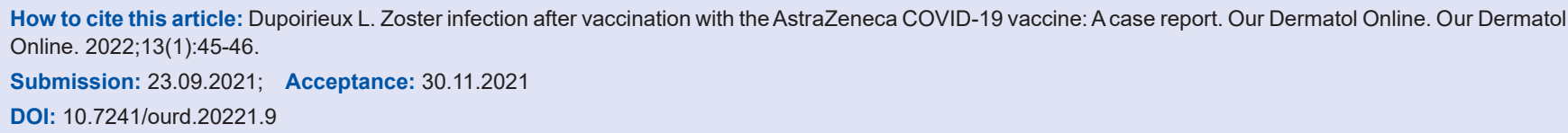




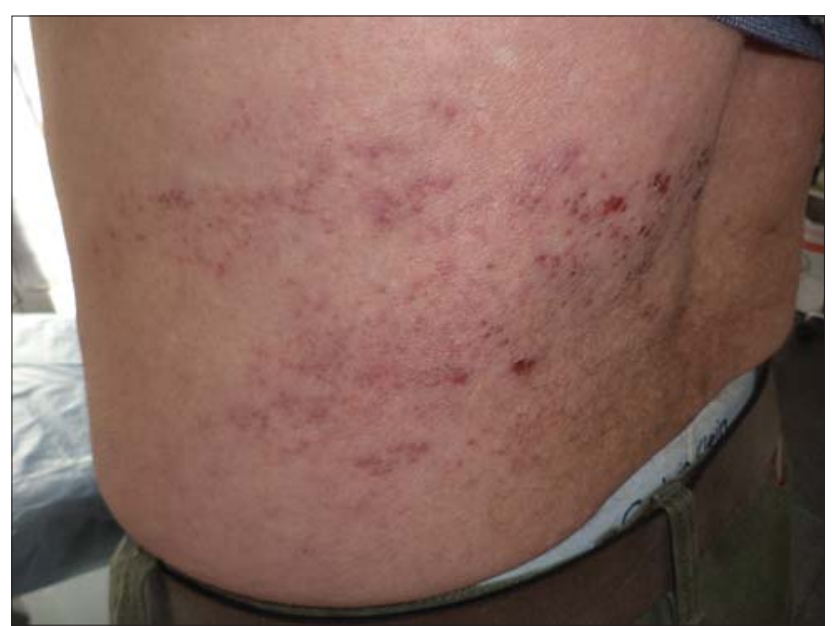

Figure 1: The eruption on day 17.

noticeable because it was covering several dermatomes, approximately from D5 to D7. Zoster reactivation after COVID-19 vaccination has now been described worldwide in the medical literature with all available vaccines (AstraZeneca, Moderna, Pfizer) [2-8]. The incidence of this adverse reaction in a Spanish study on five cases [7] was estimated to be 1995.3 cases per 100,000 after a one-month follow-up, six to seven times the estimated incidence in the general population. One intriguing fact is that three out of five patients had a positive PCR test after vaccination. However, as our patient was referred to my office for another reason, no PCR test was prescribed. The patient's recovery time was longer, yet he, fortunately, did not suffer from residual neuralgia, which seems more likely in immunocompromised patients. It has been claimed that Zoster reactivation may be caused by lymphopenia, but a biological checking displayed no problems. However, we did observe in this patient a late low level of antibodies, which raised several concerns. First, although it has been stated that a single shot of the AstraZeneca vaccine elicits a robust immunologic reaction [9], it is highly difficult to find relevant data on the long-term level of antibodies after COVID-19 vaccination. In the future, this will be of crucial importance.

Secondly, this case is embarrassing as it is now admitted that boosting immunogenicity with a second dose is absolutely essential [10]. However, we found no published guidelines concerning the administration of the second dose for patients who have experienced an adverse effect after the first jab.

\section{CONCLUSION}

Zoster reactivation is now a well-documented undesirable effect of all COVID-19 vaccines. However, it is completely unpredictable and, thus, no preventive treatment may be routinely prescribed to avoid this complication.

\section{Consent}

The examination of the patient was conducted according to the principles of the Declaration of Helsinki.

The authors certify that they have obtained all appropriate patient consent forms, in which the patients gave their consent for images and other clinical information to be included in the journal. The patients understand that their names and initials will not be published and due effort will be made to conceal their identity, but that anonymity cannot be guaranteed.

\section{REFERENCES}

1. McMahon DE, Amerson E, Rosenbach M, Lipoff JB, Moustafa D, Tyagi A, et al. Cutaneous reactions reported after Moderna and Pfizer COVID-19 vaccination: A registry-based study of 414 cases. J Am Acad Dermatol. 2021;85:46-55.

2. Aksu SB, Öztürk GZ. A rare case of shingles after COVID-19 vaccine: Is it a possible adverse effect? Clin Exp Vaccine Res. 2021;10:198-201.

3. Bostan E, Yalici-Armagan B. Herpes zoster following inactivated COVID-19 vaccine: A coexistence or coincidence? J Cosmet Dermatol. 2021;20:1566-7.

4. Channa L, Torre, K, Rothe M. Herpes zoster reactivation after mRNA-1273 (Moderna) SARS-CoV-2 vaccination. JAAD Case Rep. 2021;15:60-1.

5. Chiu HH, Wei, KC, Chen A, Wang WH. Herpes zoster following COVID-19 vaccine: Report of 3 cases. QJM 2021;22:hcab208.

6. Psichogiou M, Samarkos M, Mikos N, Hatzakis A. Reactivation of varicella zoster virus after vaccination for SARS-CoV-2 Vaccines. 2021;9:572.

7. Rodríguez-Jiménez P, Chicharro P, Cabrera LM, Seguí M, Morales-Caballero A, Llamas-Velasco M, et al. Varicella-zoster virus reactivation after SARS-CoV-2 BNT162b2 mRNA vaccination: Report of 5 cases. JAAD Case Rep. 2021;12:58-9.

8. Santovito LS, Pinna G. A case of reactivation of varicella-zoster virus after BNT162b2 vaccine second dose? Inflamm Res. 2021;970:935-7.

9. Iacobucci G. Covid-19: Most UK adults had antibodies after one dose of AstraZeneca or Pfizer vaccine, data suggest. BMJ. 2021;373:n1274.

10. Shaker M., Phillips E, Blumenthal KG, Abrams EM, Banerji A, Oppenheimer J, et al. the importance of a timely second dose of the 2021 COVID-19 mRNA vaccine depends on the protection afforded by a first dose and subsequent risk of anaphylaxis. J Allergy Clin Immunol Pract. 2021;9:2556-61.

Copyright by Laurent Dupoirieux. This is an open-access article distributed under the terms of the Creative Commons Attribution License, which permits unrestricted use, distribution, and reproduction in any medium, provided the original author and source are credited.

Source of Support: Nil, Conflict of Interest: None declared. 